\title{
Divergent topological networks in Alzheimer's disease: a diffusion kurtosis imaging analysis
}

\author{
Jia-Xing Cheng ${ }^{1}$, Hong-Ying Zhang ${ }^{2}$, Zheng-Kun Peng ${ }^{2}$, Yao Xu' ${ }^{1}$, Hui Tang ${ }^{3}$, Jing-Tao $\mathrm{Wu}^{2}$ and Jun $\mathrm{Xu}^{4,5^{*}}$
}

\begin{abstract}
Background: Brain consists of plenty of complicated cytoarchitecture. Gaussian-model based diffusion tensor imaging (DTI) is far from satisfactory interpretation of the structural complexity. Diffusion kurtosis imaging (DKI) is a tool to determine brain non-Gaussian diffusion properties. We investigated the network properties of DKI parameters in the whole brain using graph theory and further detected the alterations of the DKI networks in Alzheimer's disease (AD).

Methods: Magnetic resonance DKI scanning was performed on 21 AD patients and 19 controls. Brain networks were constructed by the correlation matrices of 90 regions and analyzed through graph theoretical approaches.

Results: We found small world characteristics of DKI networks not only in the normal subjects but also in the AD patients; Grey matter networks of AD patients tended to be a less optimized network. Moreover, the divergent small world network features were shown in the AD white matter networks, which demonstrated increased shortest paths and decreased global efficiency with fiber tractography but decreased shortest paths and increased global efficiency with other DKI metrics. In addition, AD patients showed reduced nodal centrality predominantly in the default mode network areas. Finally, the DKI networks were more closely associated with cognitive impairment than the DTI networks.
\end{abstract}

Conclusions: Our results suggest that DKI might be superior to DTI and could serve as a novel approach to understand the pathogenic mechanisms in neurodegenerative diseases.

Keywords: Small world, Alzheimer's disease, Diffusion kurtosis imaging, Brain networks

\section{Introduction}

Alzheimer's disease $(\mathrm{AD})$ is the most frequent neurodegenerative disorder causing cognitive impairment, disabilities and finally death in aged people. The upgraded guidelines recommend that the diagnosis of $\mathrm{AD}$ should be based on the psychiatric and neurological signs, imaging findings, and the presence of biomarkers [1]. Accumulated amyloid $\beta$ peptides $(A \beta)$ and phosphorylated tau have been speculated to play an early role in the brains affected by $\mathrm{AD}$ [2], followed by synaptic dysfunction, brain hypometabolism and eventually brain atrophy as the

\footnotetext{
*Correspondence: neurojun@126.com

Jia-Xing Cheng and Hong-Ying Zhang are joint first authors.

${ }^{4}$ Department of Neurology, Beijing TianTan Hospital, Capital Medical University, Beijing 100050, China

${ }^{5}$ Jiangsu Key Laboratory of Integrated Traditional Chinese and Western Medicine for Prevention and Treatment of Senile Diseases, School of

Medicine, Yangzhou University, Yangzhou 225001, Jiangsu, China

Full list of author information is available at the end of the article
}

biomarkers of neurodegeneration [3-5]. Combined Tau-A $\beta$ interactions could promote the disruption of axonal connectivity [6]. Different imaging modalities have revealed structural and functional connectivity disruptions between anatomically distinct brain regions in patients with AD. Graph theory speculates that the human brain is constructed of complex networks with topology, small world and centrality based on the measurement of structural data, diffusion tensor data and functional magnetic resonance imaging (fMRI) data [7-11]. Thus, $\mathrm{AD}$ has been proposed as a disconnection syndrome based on the converging findings.

Graph theoretical methods provide a powerful approach to quantitative analysis of the organization of network connectivity $[7-9,12-18]$. A number of characteristic properties have been used to describe the network including characteristic path length (Lp),

(C) The Author(s). 2018 Open Access This article is distributed under the terms of the Creative Commons Attribution 4.0 International License (http://creativecommons.org/licenses/by/4.0/), which permits unrestricted use, distribution, and reproduction in any medium, provided you give appropriate credit to the original author(s) and the source, provide a link to the Creative Commons license, and indicate if changes were made. The Creative Commons Public Domain Dedication waiver (http://creativecommons.org/publicdomain/zero/1.0/) applies to the data made available in this article, unless otherwise stated. 
clustering coefficient (Cp), global efficiency (Eg) and the existing hubs. The nodes of a network are represented by brain regions or voxels defined by a predetermined parcellation scheme, while the edges depend on different modalities of imaging technology [10]. Structural networks are based on brain anatomical features such as the grey matter (GM) volume, cortical thickness, surface area, and the correlations between different regional features [7, 19, 20]. Diffusion networks can be calculated by the metric of fiber number (FN), density, strength, probability, and mean diffusion measures from the data of diffusion tensor imaging (DTI) [9, 21-23].

A recent neuroimaging study has shown that small world aberrations in structural networks in AD patients were associated with $A \beta$ deposition [24]. Using graph theory analysis of the GM structural networks, both $\mathrm{He}$ et al. [7] and Yao et al. [25] revealed increased Cp and Lp of whole brain and reduced nodal centrality predominantly in the temporal-parietal association cortex regions in $\mathrm{AD}$ patients. However, Tijms et al. [17] reported an opposite finding about $\mathrm{Cp}$ and $\mathrm{Lp}$ in the cortical structural networks in AD. Previous studies also observed that white matter (WM) structural networks in both $\mathrm{AD}$ and healthy groups had a small world topology with DTI $[21,26,27]$. In addition, even in the preclinical $\mathrm{AD}$, the DTI networks were found to be impaired earlier than other structural imaging markers. Furthermore, studies showed that performance in memory and executive function in $A D$ patients were related to decreased local efficiency (Eloc), increased Lp and decreased Eg with DTI networks [9, 23, 28].

However, DTI yields only a fraction of the information potentially accessible by diffusion MRI, mainly due to the fact that the DTI is unable to quantify non-Gaussian diffusion [29]. In the brain, non-Gaussian diffusion is known to be substantial and arises from diffusion barriers, such as cell membranes and organelles as well as water-containing compartments (both extracellular and intracellular) with differing diffusion properties. In recent years, a measure of diffusional non-Gaussianity called diffusion kurtosis imaging (DKI) was developed rapidly, by which it could overcome the limitations of DTI and provide a way to investigate the microstructure of both WM and GM [30]. At least theoretically, GM tissue is of non-Gaussian distribution. The DKI model is composed of diffusion and kurtosis measurements. The kurtosis indices of mean kurtosis $(\mathrm{MK})$, axial kurtosis (AK), radial kurtosis (RK) respectively represent the average apparent kurtosis along all diffusion encoding directions, the kurtosis along the directions parallel and perpendicular to the principal diffusion direction [31, 32].
DKI has been initially applied to the study of the nervous system, such as normal brain tissue, cerebral infarction, brain tumors, brain trauma as well as Alzheimer's disease and Parkinson's disease [33-35]. For example, DKI can efficiently assess the glioma grade and cellular proliferation [36]. Up to date, few studies have applied DKI to AD patients and only revealed decreased regional kurtosis values in both the GM and WM of the parietal and occipital lobes by regions of interest measurement, and suggested that DKI could be sensitive in the assessment of microstructure damage in $\mathrm{AD}$ patients $[37,38]$. Another study indicated that the combination of diffusion and kurtosis measurements from DKI significantly yielded high performance in the pathological automatic detection of Alzheimer's disease [39]. These studies, however, have not addressed the question of whether heterogeneous DKI properties are coordinated between different cortices. More specifically, human brain WM networks can be constructed using DTI metrics for a long time and present small world, centrality and modularity. Therefore, we focus on the construction of diffusion kurtosis networks (DKN) to investigate potential aberrant mechanisms underlying brain dysfunction in AD. We hypothesize that DKN could present small world properties in the human brain, and we further investigate whether the alterations of non-Gaussian diffusion properties are sensitive to the neurodegenerative disorders such as AD.

\section{Materials and methods Subjects}

The subjects were recruited from Northern Jiangsu People's Hospital, including twenty-one $\mathrm{AD}$ patients and nineteen normal controls $(\mathrm{NC})$. The diagnosis for probably $\mathrm{AD}$ made by two experienced neurologists was based on the criteria of the National Institute of Neurological and Communicative Diseases and Stroke/Alzheimer's Disease and Related Disorders Association (NINCDS/ADRDA) [40]. Exclusion criteria were any medical, neurological, or psychiatric conditions that could account for the symptoms of dementia, and Fazekas scale of white matter hyperintensities $>2$ on brain MRI. The control group was recruited according to the distribution of the age, gender and education years in the $\mathrm{AD}$ group. All the participants were assessed referring to a standard clinical protocol, which involved the inquiry of the medical history, an interview with a spouse or close family member, blood tests, MRI of the brain according to a standard protocol and a set of neuropsychological assessments including Mini Mental State Examination (MMSE) and Montreal cognitive assessment (MoCA).

The study was approved by the Ethics Committee of Northern Jiangsu People's Hospital, Yangzhou University, and all participants or their guardians signed informed consent forms before MRI scanning. 
Images were acquired in a 3.0 T GE Discovery MR750 scanner (GE Healthcare Systems, Milwaukee, WI, USA) system with an 8-channel head coil. DKI images were acquired with $3 \mathrm{~b}$-values $\left(\mathrm{b}=0,1250\right.$ and $\left.2500 \mathrm{~s} / \mathrm{mm}^{2}\right)$ along 30 diffusion gradient directions using a single-shot EPI sequence. DKI sequence parameters were: TR $=5800 \mathrm{~ms}$, $\mathrm{TE}=100 \mathrm{~ms} ; \mathrm{FOV}=240 \times 240 \mathrm{~mm}^{2} ;$ matrix $=100 \times 100$; voxel $=2.4 \times 2.4 \times 4 \mathrm{~mm}^{3} ; 35$ axial slices. $3 \mathrm{D}$ T1WI images of the whole brain were acquired using the BRAVO sequence, the imaging parameters were: $\mathrm{TR}=8.2 \mathrm{~ms}$; $\mathrm{TE}=3$. $2 \mathrm{~ms} ; \mathrm{TI}=450 \mathrm{~ms}$; flip angle $=12^{\circ} ; \mathrm{FOV}=256 \mathrm{~mm} \times$ $256 \mathrm{~mm} ;$ matrix $=256 \times 256$; voxel $=1 \times 1 \times 1 \mathrm{~mm}^{3} ; 160$ slices.

\section{DKI data processing}

DKI data were processed by using Matlab R1202b and Diffusion Kurtosis Estimator (DKE) (http://www.nitrc. org/projects/dke) [41]. Data were first processed to correct subject motion, eddy current-induced geometric distortions and denoise. The DKI model was parameterized by the diffusion tensor (DT) and kurtosis tensor (KT) from which several rotationally-invariant scalar measures were extracted, which could be used to construct WM sensitive and GM sensitive networks respectively. The CLLS-QP algorithm used in DKE for extracting DT and KT parameters has been described in detail in the previous research [41]. The DT-derived measures included mean diffusivity (MD), axial diffusivity (Ad), radial diffusivity (RD), fractional anisotropy (FA) [42]; and KT-derived measures were AK, RK, MK and kurtosis fractional anisotropy (KFA) [41, 43]. Of the DT-derived measures, the MD corresponds to the diffusion coefficient averaged over all possible diffusion directions, whereas Ad is the diffusion coefficient in the direction of the principal diffusion tensor eigenvector and RD is the diffusion coefficient averaged over all diffusion directions perpendicular to the principal diffusion tensor eigenvector. FA measures the degree of anisotropy and ranging between 0 (fully isotropic diffusion) and 1 (fully anisotropic diffusion) [44]. The data were used in fiber tracking (FT) module embedded in DKE to get the outputs for further use.

\section{Cortical parcellation}

Each individual high-resolution structural image (T1 image) was first coregistered to the FA image in the diffusion space using a linear transformation. The transformed structural image was then mapped to the T1 template of the Montreal Neurological Institute (MNI) space using a nonlinear transformation [45]. The resulting inverse transformation was then used to warp the Automated Anatomical Labeling (AAL) mask from the MNI space to the individual native space.

\section{Network construction}

GM network was defined as a correlation network based on each DKI parameter measurement. A regional parameter was computed as the average value of all voxels within the region. The interregional correlation matrix of each group was then obtained by calculating the correlation coefficients across individuals between the regional parameters of every pair of regions using Pearson's correlation. Therefore, AD and control groups achieved a parameter matrix for each DKI metric respectively. The grey matter network contained $\mathrm{N}$ nodes and $\mathrm{K}$ edges, which included 90 cortical and sub-cortical regions in the AAL atlas (45 for each hemisphere) [46], the parameter correlation value between the two regions across all subjects meant the $K$ values.

To construct DKI WM network, the brain nodes were also defined by the AAL atlas. Each edge represented the connecting fiber that linked a couple of brain nodes. The weights of the edges were defined as FN, mean fiber length and mean diffusion measures, including FA, MD, Ad, RD, MK, RK, AK and KFA. All the image processes of kurtosis diffusion network construction were manipulated using PANDA (http://www.nitrc.org/projects/ panda) [47].

Each correlation matrix was thresholded over a wide range of sparsity $(6 \%-40 \%)$, and the properties of the resulting graphs at each threshold value were estimated. Subsequent indicators were calculated in the Gretna (http://www.nitrc.org/projects/gretna/) [48].

\section{Small world analysis}

Small world measures of a network ( $\mathrm{Cp}$ and $\mathrm{Lp}$ ) were originally proposed by Watts and Strogatz [49]. For both KT and DT networks, we calculated the Cp (the number of existing connections among the neighbors of the node divided by all their possible connections), Lp (the average minimum number of connections that link any two nodes of the network), and betweenness centrality (the number of the shortest paths between any two nodes that run through a node). A real network was defined as small world if it met the following criteria: $\gamma=\mathrm{Cp} /$ mean $($ Cprand $)>1$ and, $\lambda=\mathrm{Lp} /$ mean(Lprand) $\approx 1 \quad$ [49], where the Cprand and Lprand were the mean $\mathrm{Cp}$ and Lp of matched random networks that preserve the same number of nodes and edges as the real network $[50,51]$.

\section{Statistical analysis}

The significance threshold was set top $\leq 0.05$ for all analyses. All continuous variables were tested for normal distribution within groups using the Kolmogorov-Smirnov test. Differences between AD subjects and NC regarding demographical data (age, sex), MMSE and MoCA scores were calculated as follows: the chi-square test was used 
for categorical variables; the t-test was used for continuous normally distributed variables.

To determine the differences in GM graph network parameters between groups, a nonparametric permutation test method was used [52]. First, $\mathrm{Cp}$ and Lp of the networks at a given sparsity were computed separately for the $\mathrm{AD}$ and control groups. To test the null hypothesis that the observed group differences could occur by chance, we then randomly reallocated each subject's set of regional mean kurtosis measures to one or the other of the two groups and recomputed the correlation matrix for each randomized group. We then obtained corresponding weighted matrix using the same sparsity threshold as in the real brain networks. Next, we calculated the network parameters for each randomized group and obtained their differences between the randomized groups. This randomization procedure was repeated 1000 times.

To determine the between-group differences in the small world properties and network efficiency of the WM networks, an analysis of covariance (ANCOVA) was performed on each diffusion metric. Age and gender were taken as covariates in this model. The relationship between the network metrics and MMSE and MoCA scores in the patient group was analyzed by the partial correlation analysis.

\section{Results}

\section{Demographics}

There were no significant differences in both age $(p=0$. $07)$ and gender $(p=0.8)$ of $\mathrm{AD}$ patients and controls. For the neuropsychological tests, there were significant differences in MMSE and MoCA scores between the two groups $(\mathrm{p}<0.05$, Table 1$)$.

\section{Within group network analysis}

\section{Small world analysis of the grey matter networks}

The interregional parameter correlation values of the cortical networks were calculated to construct correlation matrices $(90 \times 90)$ for the $\mathrm{NC}$ and AD groups. The images of the group level interregional correlation matrices using DKI metrics of MK, KFA, AK and RK are shown in Fig. 1, and KFA in the control group presented the strongest positive coordinated effects during observations among these metrics.

Table 1 Demographic and clinical characteristics of subjects

\begin{tabular}{llll}
\hline Characteristics & AD Patients & Controls & $P$ value \\
\hline Age & $73.57( \pm 6.87)$ & $70( \pm 8.08)$ & 0.07 \\
Female/male & $12 / 21$ & $9 / 18$ & 0.8 \\
MMSE & $18.71( \pm 5.9)$ & $28.16( \pm 1.12)$ & $<0.001$ \\
MoCA & $15( \pm 6.67)$ & $27.89( \pm 0.14)$ & $<0.001$ \\
\hline
\end{tabular}

Scores are shown with mean $( \pm \mathrm{SD})$

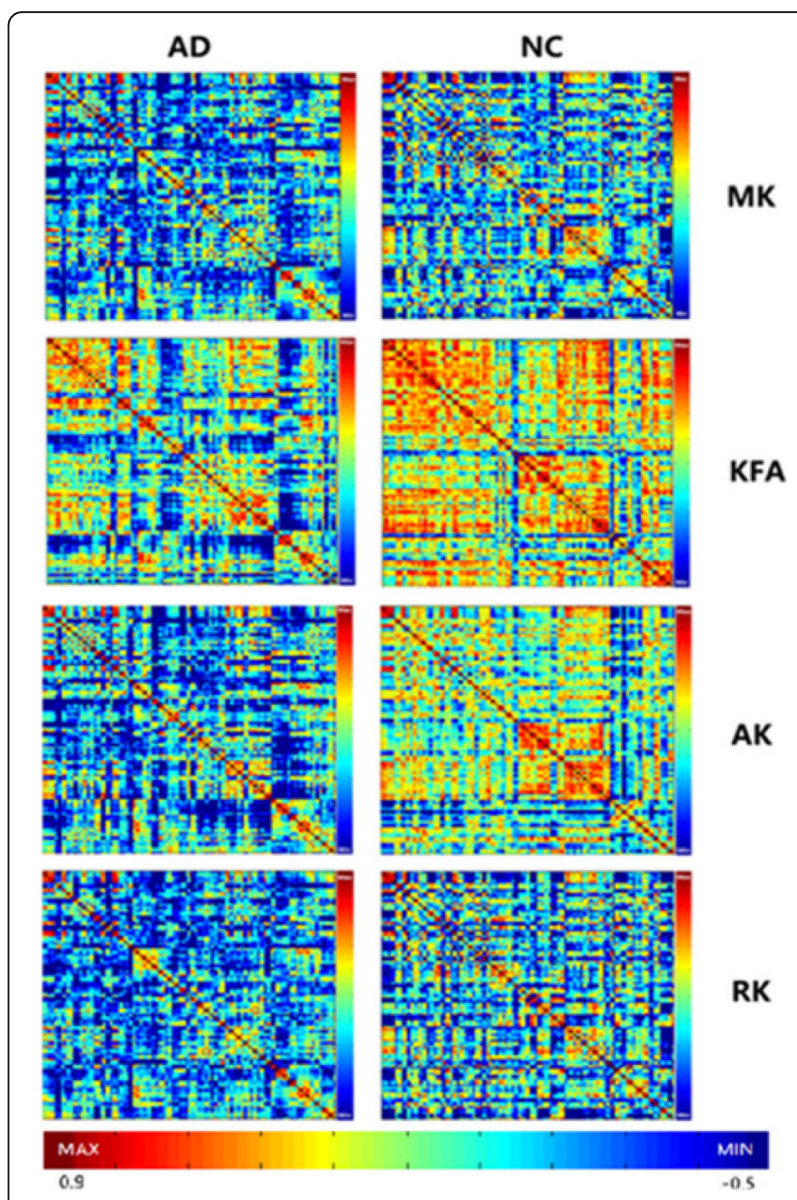

Fig. 1 The interregional correlation matrix $(90 \times 90)$ in the $A D$ and NC groups using DKI metrics of MK, KFA, AK and RK. The color bar indicates the value of the interregional parameter correlation. The red color bar represents the higher positive correlation value. The blue color bar represents the higher negative correlation value. From the maps, a great degree of dispersion in DKI could be observed in AD patients. Note more strong positive coordinated effects existing in extensive brain regions labeled by red color for the metric of MK, KFA, AK and RK in the control group vs. AD group, and KFA is the typical. The higher KFA value meant more compact histological structure

We found the small world attributes of GM networks with MK, KFA, AK and RK metric in the normal elder subjects and $\mathrm{AD}$ patients over a wide range of sparsity (6\% $\sim \mathrm{S} \sim 40 \%)$ (showed in Fig. 2). The small-worldness values $(\sigma=\gamma / \lambda)$ calculated from the DKI indices were larger than 1, the values with MK, KFA, AK and RK were $1.57,1.67,1.75$ and 2.25 in $\mathrm{AD}$ patients, respectively, and were $2.88,2.28,1.55,2.24$ in controls, respectively.

\section{Small world analysis of the white matter networks}

As expected, we also observed that the brains of both the AD patients and controls had prominent small world network properties in WM networks over a wide range 

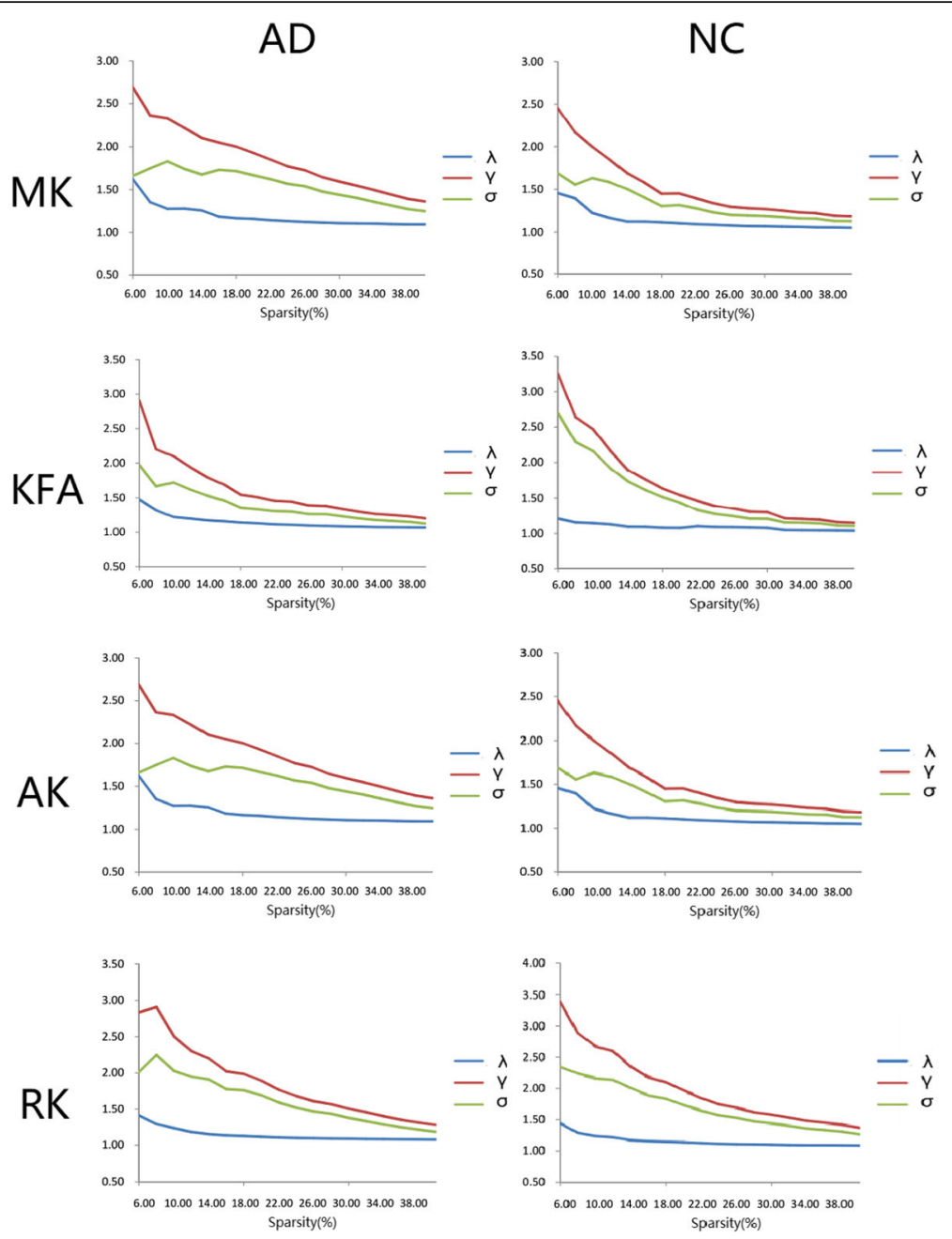

Fig. 2 Small world properties of GM networks in the AD and NC using DKI metrics of MK, KFA, AK and RK. Both networks demonstrated small world architectures over a wide range of sparsity $(6 \% \sim S \sim 40 \%)$ in comparison with the matched random networks. $\gamma$ value was calculated by $\mathrm{Cp} /$ mean (Cprand), $\lambda$ value was calculated by Lp/mean (Lprand). The small-worldness value of $\sigma$ was presented by $\gamma / \lambda$

of sparsity $(6 \% \sim \mathrm{S} \sim 40 \%)$ in comparison with the matched random networks. The small-worldness values calculated from all DKI indices were larger than 1, the values with FA, MD, Ad, RD, MK, RK, AK and KFA were $1.57,1.50,1.28,1.54,2.04,1.65,2.96,1.70$ in $\mathrm{AD}$ patients, respectively, and were $1.32,1.57,1.32,1.76,1$. $47,1.33,1.72,1.43$ in controls, respectively (Table 2). The small-worldness values constructed by FN were 3 . 02 in $\mathrm{AD}$ patients and 1.84 in controls.

\section{Hub region analysis of the grey matter networks}

To identify the hub regions, we examined normalized nodal betweenness centrality for the $M K$ metric $(b i, b i=B i / B$, where $\mathrm{B}$ was the average betweenness of the network. bi was a global centrality measure that captured the influence of a node over information flow between other nodes in the network) of each cortical region in both groups. When the betweenness value of a node was more than 1.5 times the average betweenness of the network ( $\mathrm{bi}>1.5$ ), the node was considered as a hub [53]. In the control group, 10 regions including 4 association cortex regions and 6 paralimbic cortex regions were identified as the hubs (Table 3), and in the $\mathrm{AD}$ group, 9 association cortex regions were identified as the hubs (Table 4). These identified hubs were predominately located in regions of association cortex such as superior temporal gyrus, middle temporal gyrus, middle frontal gyrus and fusiform gyrus (Fig. 3), and compared to the controls, less hub regions were observed in thalamus and paralimbic system in $\mathrm{AD}$ patients, which were mostly involved in default mode.

\section{Between group network analysis Comparison in grey matter networks}

For the MK metric of DKI networks over a wide range of sparsity, AD patients showed increased Lp and decreased Cp compared with controls (showed in Fig. 4). 
Table 2 Comparison of DKI metrics networks between control and AD group

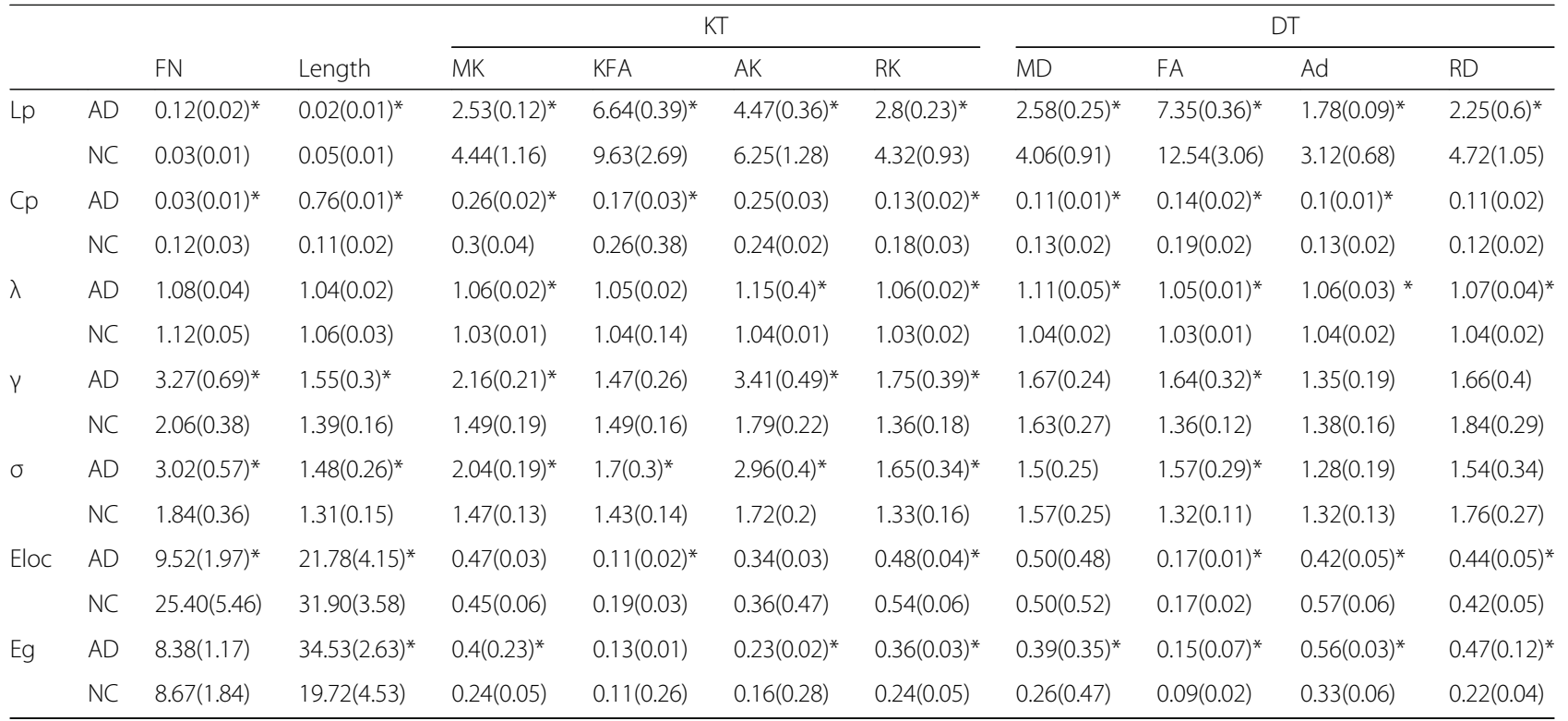

${ }^{*} p<0.05$, corrected. $\gamma$ value was calculated by $\mathrm{Cp} /$ mean(Cprand), $\lambda$ value was calculated by $\mathrm{Lp} / \mathrm{mean}$ (Lprand). The small-worldness value of $\sigma$ was presented by $\gamma / \lambda$. Scores are shown with mean $( \pm \mathrm{SD})$

Permutation test further revealed significant differences $(\mathrm{p}<0.05)$ in the $\mathrm{Lp}$ values at $10 \%<\mathrm{S}<40 \%$ and $\mathrm{Cp}$ values at $14 \%<\mathrm{S}<40 \%$.

\section{Comparison in white matter networks}

We constructed WM networks by using diffusion probabilistic tractography, and compared with controls, networks of FN in AD patients showed significantly increased Lp and decreased Eg (Fig. 5). For the WM networks with the DKI

Table 3 Regions showing high betweenness centrality in DKN of control subjects

\begin{tabular}{lll}
\hline Regions & Class & $\begin{array}{l}\text { Normalized } \\
\text { betweenness, bi }\end{array}$ \\
\hline $\begin{array}{l}\text { Left inferior frontal gyrus, } \\
\text { opercular part }\end{array}$ & Paralimbic & 4.69 \\
$\begin{array}{l}\text { Right precuneus } \\
\text { Left hippocampus }\end{array}$ & Paralimbic & 4.40 \\
$\begin{array}{l}\text { Right inferior frontal gyrus, } \\
\text { opercular part }\end{array}$ & Paralimbic & 4.26 \\
$\begin{array}{l}\text { Left superior frontal gyrus, } \\
\text { orbital part }\end{array}$ & Paralimbic & 4.05 \\
$\begin{array}{l}\text { Left fusiform gyrus } \\
\text { Left superior temporal gyrus }\end{array}$ & Paralimbic & 3.40 \\
Right fusiform gyrus & Association & 3.30 \\
Left thalamus & Association & 2.79 \\
Right inferior frontal gyrus, & Association & 2.67 \\
triangur paralimbic & 2.24 \\
\end{tabular}

triangular part

The hub regions (bi>1.5) in the GM network of the control group for the MK metric were listed in a descending order of their normalized betweenness, bi. The regions were classified as primary, association and paralimibic as described by Mesulam (1998) metrics of MK, AK, RK, KFA, FA, MD, RD and Ad, AD patients showed significantly decreased Lp compared with controls ( $<<0.05$, corrected). Most of the DKI metrics showed decreased $\mathrm{Cp}$ except AK and RD in AD $(\mathrm{p}<0.05$, corrected). For efficiency measurements of the WM networks, the Eloc and Eg were computed. Compared with the controls, no significant alterations in the Eloc were observed in $\mathrm{AD}$ patients, whereas significantly increased $\mathrm{Eg}$ was found using MK, AK and MD metrics. The differences in network metrics were listed in Table 2. These findings indicated that distinct small world network properties could be derived from different $\mathrm{DKI}$ indices in $\mathrm{AD}$ patients.

\section{Comparison of small word attributions between KT and DT networks}

As shown in Table 2, KT and DT small world attributes (Lp, Cp, $\lambda, \gamma$ and $\sigma$ ) in AD were listed. We found that AD patients had increased $\sigma$ value for all KT-derived indices and merely FA of DT-derived indices. Similarly, MK and RK from KT-derived indices showed significant differences concurrently in the small world attributes of $\mathrm{Lp}, \mathrm{Cp}, \lambda$ and $\gamma$, in contrast to only FA from DT-derived indices.

\section{Relationship of small world networks and cognitive performance}

We next examined the relationships between the network metrics (small world and efficiency metrics) and cognitive performance. Partial correlation analysis with age and gender as confounding covariates were separately performed for the AD group. The results showed that KT metrics had closer ties with cognitive functions 
Table 4 Regions showing high betweenness centrality in DKN of $A D$ patients

\begin{tabular}{lll}
\hline Regions & Class & $\begin{array}{l}\text { Normalized } \\
\text { betweenness,bi }\end{array}$ \\
\hline Right superior temporal gyrus & Association & 6.87 \\
Right inferior temporal gyrus & Association & 3.96 \\
Right middle temporal gyrus & Association & 3.62 \\
Left middle temporal gyrus & Association & 3.52 \\
Right temporal pole, superior & Paralimbic & 3.44 \\
temporal gyrus & & \\
Left insula & Association & 3.19 \\
Left heschl gyrus & Association & 3.04 \\
Right fusiform gyrus & Association & 2.86 \\
Left Inferior parietal, but & Association & 2.64 \\
supramarginal and angular gyri & & \\
Left middle frontal gyrus & Association & 2.55
\end{tabular}

The hub regions (bi>1.5) in the GM network of the AD group for the MK metric were listed in a descending order of their normalized betweenness, bi. The regions were classified as primary, association and paralimibic as described by Mesulam (1998)

than DT metrics, particularly for FN, MK, KFA and AK metrics $(p<0.05$, corrected. See Table 5$)$. We found that MMSE scores were significantly positively correlated with Lp of MK, $\lambda$ of RK as well as Eloc of FN, KFA and RK, while negatively correlated with $\sigma$ of FN, MK and KFA. In addition, MoCA scores were significantly positively correlated with $\mathrm{Lp}$ of $\mathrm{AK}, \lambda$ of fiber length, RK, $\mathrm{MD}$, as well as Eloc of FN, RD and AK, while negatively correlated with $\sigma$ of FN, MK and Eg of AK.

\section{Discussion}

Our current study investigated DKN in AD patients and healthy controls. To our best knowledge, no previous report on the construction of DKI networks has so far been published. The main findings are as follows: (1) DKN presented small world characteristics in the brains of both healthy subjects and AD patients. (2) GM network of $\mathrm{AD}$ patients tended to be a less optimized network. (3) AD WM networks showed increased Lp and decreased $\mathrm{Eg}$ for FN, by contrast, decreased Lp and increased Eg for the other diffusion metrics of MK, RK, AK, KFA, FA, MD, RD and Ad. (4) Regional hubs decreased in AD. (5) Kurtosis parameters had stronger associations with cognitive impairment in $\mathrm{AD}$ patients than the DT-derived parameters.

Alterations of small world topological properties in $\mathrm{AD}$ have been demonstrated by using different modalities such as Electroencephalogram (EEG), Magnetoencephalogram (MEG), fMRI, structural magnetic resonance imaging (sMRI) and DTI in plenty of previous studies. However, there are inconsistent findings about the small world properties. Most structural connectivity networks exhibited increased Lp in AD-related networks. For example, by sMRI and DTI network analysis, Cp and Lp were showed significantly increased in $\mathrm{AD}$ patients compared to the controls $[7,9]$. On the contrary, decreased Lp in AD was also demonstrated by using sMRI and DTI [17, 54]. For functional connectivity network analysis, EEG and fMRI studies showed that AD patients had increased Lp $[8,55]$. In contrast, decreased Lp was found in brain functional networks of $\mathrm{AD}$ patients using
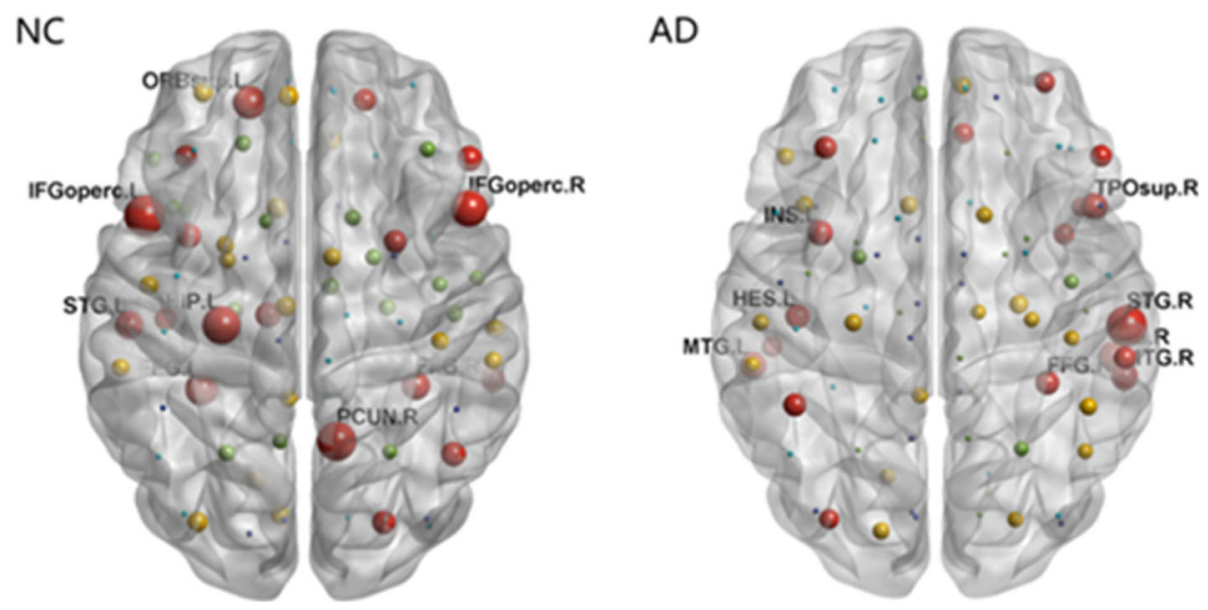

Fig. 3 Hub regions within groups for MK metric networks. Each ball represented corresponding brain region in AAL atlas, displayed in the center of the region. The size of balls represented the bi value. Only the hub regions with bi>1.5 were indicated by red. Note the hub regions in $A D$ became sparse in the default mode areas. The figure was processed by BrainNet Viewer software. IFGoperc.L: Left inferior frontal gyrus, opercular part; PCUN.R: Right precuneus; IFGoperc.R: Right inferior frontal gyrus, opercular part; STG.L: Left superior temporal gyrus; STG.R: Right superior temporal gyrus; FFG.L: Left fusiform; FFG.R: Right fusiform; ORB.sup.L: Left superior frontal gyrus, medial orbital; HIP.L: Left hippocampus; MTG.L: Left middle temporal gyrus; MTG.R: Right middle temporal gyrus; TPOsup.R: Right temporal pole, superior temporal gyrus; INS.L: Left insula; HES.L: Left heschlgyrus 

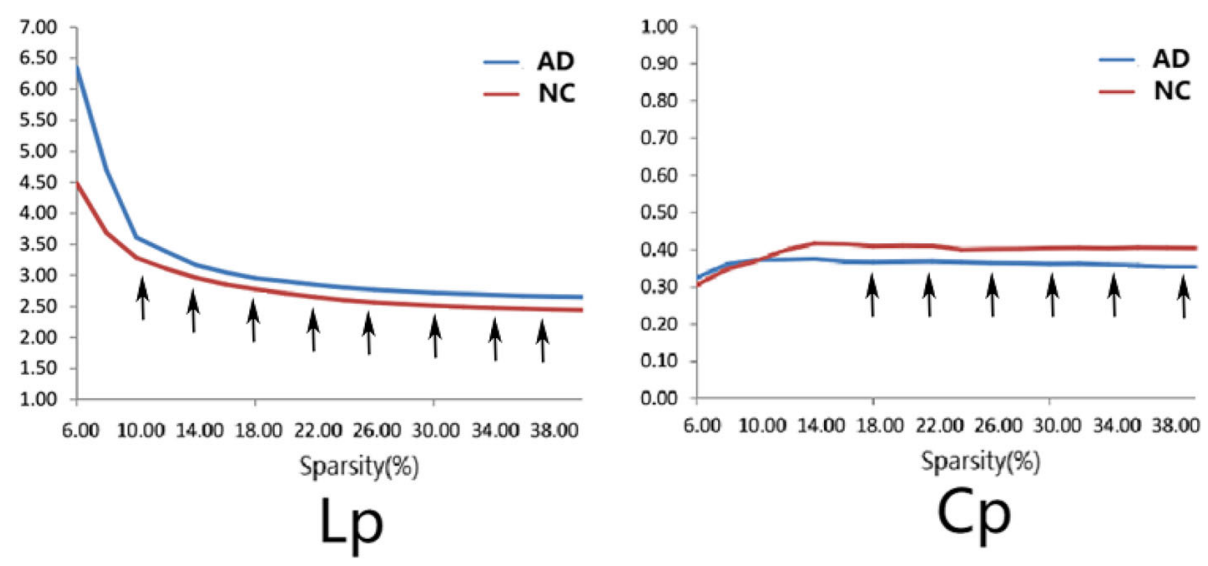

Fig. 4 Between-group grey matter differences in $L p$ and $C p$ properties. The graph shows AD patients (blue lines) presented with increased Lp values and decreased $\mathrm{Cp}$ values in the brain networks than controls (red lines) over a wide range of thresholds. Statistical analysis of the between-group differences obtained by 1000 permutation tests further revealed significant differences $(p<0.05)$ in the $L p$ values at $10 \%<S<40 \%$ and $\mathrm{Cp}$ values at $14 \%<\mathrm{S}<40 \%$ (the black arrows)

fMRI [56], EEG [57] and MEG [58]. The discrepancies in the brain network analysis could be due to different modalities. Our findings with DKI networks could integrate the discrepancies.

\section{Divergent features in WM network metrics}

The connectivity weights of the white matter networks were defined with the DT and KT metrics that represented distinct biological significance. The weighted networks retain the biophysical information in the graph model. MK reflects the general tissue complexity and the lesion heterogeneity [59]. AK is mainly affected by the cellular structure, and RK is mainly affected by the cell membrane and the myelin sheath [60]. KFA is mathematically analogous to FA, and provides complementary information about anisotropy in diffusion dynamics to describe deep brain structures [61]. The higher KFA value means more compact histological structure. In our findings of KFA matrixes, reduced coordinated effects between the extensive brain regions in $\mathrm{AD}$ meant loosened microstructure. The Ad might assess the integrity of axonal conduction. RD might be an adequate parameter for the assessment of myelin integrity [62].

In our findings, divergent features in WM network alterations presented in $\mathrm{AD}$ brains with increased $\mathrm{LP}$ and decreased Eg for FN, on the contrary, for most of the diffusion metrics such as MK, RK, AK, KFA, FA, $\mathrm{MD}, \mathrm{RD}$ and Ad with decreased Lp and increased $\mathrm{Eg}$
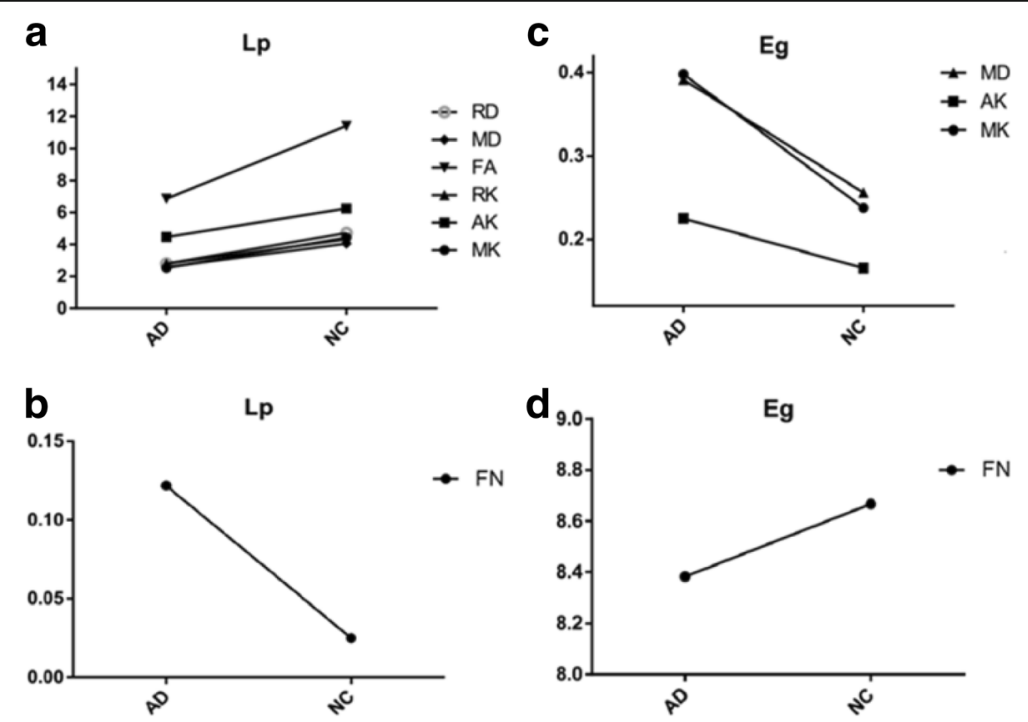

Fig. 5 Comparison of small world properties in the WM networks between AD and NC group. a. Lp values of DKI metrics; b. LP values of FN; c. Eg of DKI metrics; $\mathbf{d}$. Ad: Eg of FN. Divergence network features in Lp and Eg were displayed by various DKI parameters 
Table 5 Partial correlation analysis between small world DKI networks and cognitive performances in AD patients

\begin{tabular}{|c|c|c|c|c|c|c|c|c|c|c|c|}
\hline & & & & & & & & & & & \\
\hline & & FN & Length & $\overline{M K}$ & KFA & $A K$ & RK & $\mathrm{MD}$ & FA & $\mathrm{Ad}$ & $\mathrm{RD}$ \\
\hline$L p$ & MMSE & 0.01 & -0.18 & $0.33^{*}$ & 0.20 & 0.50 & 0.46 & -0.06 & 0.06 & -0.04 & -0.07 \\
\hline & MOCA & 0.20 & -0.09 & 0.25 & -0.07 & $0.58^{*}$ & 0.41 & -0.13 & -0.09 & -0.07 & -0.03 \\
\hline $\mathrm{Cp}$ & MMSE & 0.16 & -0.75 & -0.11 & 0.11 & -0.03 & -0.08 & 0.37 & 0.20 & -0.14 & 0.11 \\
\hline & MOCA & 0.11 & -0.70 & -0.06 & 0.20 & $-0.03^{*}$ & -0.12 & 0.40 & 0.21 & -0.16 & 0.13 \\
\hline$\lambda$ & MMSE & -0.42 & 0.77 & -0.28 & -0.43 & 0.22 & $0.48^{*}$ & 0.08 & $-0.06^{*}$ & -0.40 & 0.24 \\
\hline & MOCA & -0.43 & $0.70^{*}$ & -0.25 & 0.30 & 0.29 & $0.41^{*}$ & $0.09^{*}$ & -0.15 & -0.53 & 0.27 \\
\hline Y & MMSE & $0.43^{*}$ & -0.76 & $0.19^{*}$ & $0.45^{*}$ & -0.20 & -0.46 & -0.09 & 0.07 & 0.41 & -0.26 \\
\hline & MOCA & $0.45^{*}$ & -0.70 & 0.36 & -0.08 & -0.27 & -0.39 & -0.10 & 0.19 & 0.55 & -0.29 \\
\hline$\sigma$ & MMSE & $-0.44^{*}$ & 0.73 & $-0.19^{*}$ & $-0.45^{*}$ & 0.21 & 0.46 & 0.09 & -0.07 & -0.42 & 0.26 \\
\hline & MOCA & $-0.45^{*}$ & 0.65 & $-0.30^{*}$ & $0.08^{*}$ & 0.27 & 0.38 & 0.10 & -0.19 & -0.56 & 0.29 \\
\hline Eloc & MMSE & $0.22^{*}$ & 0.58 & $0.20^{*}$ & $0.21^{*}$ & $-0.11^{*}$ & -0.07 & -0.30 & -0.03 & -0.08 & -0.02 \\
\hline & MOCA & $0.21^{*}$ & 0.62 & 0.16 & -0.33 & $0.19^{*}$ & 0.08 & -0.30 & -0.02 & -0.17 & $0.06^{*}$ \\
\hline $\mathrm{Eg}$ & MMSE & 0.02 & $-0.37^{*}$ & 0.37 & 0.11 & $0.50^{*}$ & 0.47 & -0.11 & 0.07 & -0.03 & -0.08 \\
\hline & MOCA & 0.11 & $-0.35^{*}$ & 0.31 & 0.20 & $0.58^{*}$ & 0.42 & -0.18 & -0.07 & -0.06 & -0.05 \\
\hline
\end{tabular}

The partial correlations were computed with age and gender as the confounding covariates. ${ }^{*} p<0.05$, corrected. $\gamma$ value was calculated by $\mathrm{Cp} / \mathrm{mean}(\mathrm{Cprand}), \lambda$ value was calculated by $L p /$ mean(Lprand). The small-worldness value of $\sigma$ was presented by $\gamma / \lambda$. Note there are more KT metrics exhibiting significant correlations to cognitive performances than DT metrics

findings. In addition, most of the DKI metrics showed significant decreases in $\mathrm{Cp}$ in the AD group. Increased Lp implied the diminished information propagation ability of the fiber tract, and similar findings were demonstrated in previous studies using DTI and fMRI $[8,9,63,64]$. For the observation of decreased Lp and increased Eg, we speculated that it could result from progressive dedifferentiation among different domain-specific cortical regions in AD brains. This kind of dedifferentiation tendency has been revealed in normal aging people and $\mathrm{AD}$ patients. For example, age-dependent epigenetic assimilation was correlated with increased similarity between the cerebral cortex and the cerebellum, indicating potential brain cell dedifferentiation [65]. Different networks were also observed to merge with aging by fMRI measurements from a macro perspective [66].

We assumed that the index of FN and the other sides of DKI reflected different aspects of the brain. DKI could quantify the deviation of water molecule diffusion from the Gaussian distribution in contrast to DTI Gaussian model. All the DKI metrics reflect microstructure complexity from various diffusion models, and when damaged, for example due to deposition of amyloid plaques and tangles, the wellorganized and complex microstructures changed as well as the relevant kurtosis value [39]. However, this abnormally changing direction was not determinate, leading to divergent effects as our findings showed.

\section{Disruption of grey matter networks}

In contrast to most diffusion MRI studies that focus merely on white matter networks, our study paid attention to changes in both GM and WM networks. In our work, we constructed GM network based on each DKI parameter measurement and found small word properties, which is consistent with previous studies based on cortical thickness [7]. The alterations of increased $\mathrm{Lp}$ and decreased $\mathrm{Cp}$ in $\mathrm{AD}$ indicated a less optimized network. We assumed the damage of smallworldness in $\mathrm{AD}$ patients was due to the changes in the tissue microstructure, and could be caused by astrogliosis, microglial activation, vascular hyalinization and axonal loss in the pathology of AD.

\section{Comparison between DKI and DTI}

We contrasted the KT- with DT-derived networks. Firstly, our findings revealed impairment in grey matter networks by KT-derived metrics in AD patients, which is not assessable with DT-derived indices. Secondly, for the betweengroup comparison in the white matter networks (Table 2), all KT-derived indices, contrast to merely FA in DTderived indices, demonstrated sensitivity to the AD brains with significantly increased small-worldness values $(\sigma)$. FA has been a commonly used metric during the construction of DTI networks, and FA was considered as a biomarker of axonal integrity in the past. What's more, our findings provided additional diffusion indices sensitive to the degenerative brain impairment. We considered that MK and RK could be optimal choices to describe the white matter networks. Evidence in previous studies indicated that MK was sensitive to demyelination, axonal damage and loss, compared to DTI analysis $[67,68]$. RK might capture the diffusion heterogeneity arising from axonal membranes 
and myelination, may also potentially serve as a more sensitive marker in white matter $[60,62]$. Finally, the alterations of diffusion kurtosis network showed a close association with the cognitive performance in our findings. More specifically, kurtosis parameters of MK, AK and KFA had stronger correlations with the cognitive performance in $\mathrm{AD}$ patients than the DT-derived analogical parameters.

Our findings suggested the wide range of pathological detection of kurtosis might provide more information than diffusion tensor by determining intrinsic microstructural properties with non-Gaussian measurements.

\section{Hub changes of small world networks}

Hub nodes play an important role in transferring information between different parts of the brain network, which determines the integration efficiency of the neural information and the stability of the network structure in the networks. Our results showed that hub regions were mostly involved in the default mode in the control group, which were consistent with the previous work [69]. In contrast, hub regions within the DKI networks diminished in the AD group. Previous studies have demonstrated that loss of connectivity in default network is a possible mechanism of AD brain damage [24, 70, 71]. In addition, we found that some hub regions supervising language were impaired, including inferior frontal operculum and superior temporal gyrus.

Our study has some limitations. At first, our sample size is limited. Secondly, the exact relationship between varied indices of DKI and the complexity of brain remains not entirely clear. The reliability and reproductivity of the DKI networks remain to be confirmed in further studies.

\section{Conclusions}

We observed interesting small world properties with DKI in human brain and impaired small world networks in the AD pathology, and divergent WM network features were demonstrated in $\mathrm{AD}$ brains. In addition, the damage of DKI networks was associated with cognitive performance. DKI parameters might have the superiority to DTI and could be used as a neuroimaging marker for $\mathrm{AD}$ by characterizing the microstructure of WM and GM.

\footnotetext{
Abbreviations

AD: Alzheimer's disease; Ad: axial diffusivity; AK: axial kurtosis; Cp: clustering coefficient; DKE: Diffusion Kurtosis Estimator; DKI: diffusion kurtosis imaging; DKN: diffusion kurtosis networks; DT: diffusion tensor; DTI: diffusion tensor imaging; Eg: global efficiency; Eloc: local efficiency; FA: fractional anisotropy; FN: fiber number; FT: fiber tracking; KFA: kurtosis fractional anisotropy; KT: kurtosis tensor; Lp: characteristic path length; MD: mean diffusivity; MK: mean kurtosis; MMSE: Mini Mental State Examination; MoCA: Montreal cognitive assessment; RD: radial diffusivity; RK: radial kurtosis; sMRI: structural magnetic resonance imaging
}

\section{Acknowledgements}

We thank Prof. Gao-Lang Gong and Dr. Chen-Xi Zhao from State Key Laboratory of Cognitive Neuroscience and Learning, Beijing Normal University for assistance in data analysis.

\section{Funding}

This work was supported by the National Natural Science Foundation of China (Grants NO. 81471642, 81571652, 81271211, 81471215), Natural Science Foundation of Jiangsu Province (Grants NO.BK20151592) and Jiangsu social development project (Grants NO.BE2015665).

\section{Availability of data and materials}

Please contact author for data requests.

\section{Authors' contributions}

JX, H-YZ, YX and J-TW contributed study concept and design. J-XC, Z-KP and HT acquired, analyzed or interpreted data. J-XC, JX and H-YZ drafted and revised the manuscript. All the authors approve the final manuscript.

\section{Ethics approval and consent to participate}

The study was approved by the Ethics Committee of Northern Jiangsu People's Hospital, Yangzhou University, with informed consent provided by all participants.

\section{Competing interests}

The authors declare that they have no competing interests.

\section{Author details}

'Department of Neurology, Northern Jiangsu People's Hospital, Yangzhou University, Yangzhou 225001, China. ${ }^{2}$ Department of Radiology, Northern Jiangsu People's Hospital, Yangzhou University, Yangzhou 225001, China. ${ }^{3}$ Medical Experimental Center, Northern Jiangsu People's Hospital, Yangzhou University, Yangzhou 225001, China. ${ }^{4}$ Department of Neurology, Beijing TianTan Hospital, Capital Medical University, Beijing 100050, China. ${ }^{5}$ Jiangsu Key Laboratory of Integrated Traditional Chinese and Western Medicine for Prevention and Treatment of Senile Diseases, School of Medicine, Yangzhou University, Yangzhou 225001, Jiangsu, China.

Received: 22 January 2018 Accepted: 10 April 2018

Published online: 27 April 2018

\section{References}

1. Sperling RA, Aisen PS, Beckett LA, Bennett DA, Craft S, Fagan AM, Iwatsubo $\mathrm{T}$, Jack CR Jr, Kaye J, Montine TJ, et al. Toward defining the preclinical stages of Alzheimer's disease: recommendations from the National Institute on Aging-Alzheimer's Association workgroups on diagnostic guidelines for Alzheimer's disease. Alzheimers Dement. 2011;7(3):280-92.

2. Jack CR Jr, Holtzman DM. Biomarker modeling of Alzheimer's disease. Neuron. 2013;80(6):1347-58.

3. Delbeuck X, Collette F, Van der Linden M. Is Alzheimer's disease a disconnection syndrome? Neuropsychologia. 2007;45(14):3315-23.

4. Arendt T. Synaptic degeneration in Alzheimer's disease. Acta Neuropathol. 2009;118(1):167-79.

5. Takahashi RH, Capetillo-Zarate E, Lin MT, Milner TA, Gouras GK. Cooccurrence of Alzheimer's disease ss-amyloid and tau pathologies at synapses. Neurobiol Aging. 2010;31(7):1145-52.

6. Pooler AM, Polydoro M, Wegmann SK, Pitstick R, Kay KR, Sanchez L, Carlson GA, Gomez-Isla T, Albers MW, Spires-Jones TL, Hyman BT. Tau-amyloid interactions in the rTgTauEC model of early Alzheimer's disease suggest amyloid-induced disruption of axonal projections and exacerbated axonal pathology. J Comp Neurol. 2013;521(18):4236-48.

7. He Y, Chen Z, Evans A. Structural insights into aberrant topological patterns of large-scale cortical networks in Alzheimer's disease. J Neurosci. 2008; 28(18):4756-66

8. Sanz-Arigita EJ, Schoonheim MM, Damoiseaux JS, Rombouts SARB, Maris E, Barkhof F, Scheltens P, Stam CJ. Loss of 'small-world' networks in Alzheimer's disease: graph analysis of fMRI resting-state functional connectivity. PLoS One. 2010;5(11):e13788.

9. Lo CY, Wang PN, Chou KH, Wang J, He Y, Lin CP. Diffusion tensor tractography reveals abnormal topological organization in structural cortical networks in Alzheimer's disease. J Neurosci. 2010;30(50):16876-85. 
10. Tijms BM, Wink AM, de Haan W, van der Flier WM, Stam CJ, Scheltens P, Barkhof F. Alzheimer's disease: connecting findings from graph theoretical studies of brain networks. Neurobiol Aging. 2013;34(8):2023-36.

11. Teipel S, Drzezga A, Grothe MJ, Barthel H, Chételat G, Schuff N, Skudlarski P, Cavedo E, Frisoni GB, Hoffmann W, et al. Multimodal imaging in Alzheimer's disease: validity and usefulness for early detection. The Lancet Neurology. 2015;14(10):1037-53

12. Ciftci K. Minimum spanning tree reflects the alterations of the default mode network during Alzheimer's disease. Ann Biomed Eng. 2011;39(5):1493-504.

13. de Haan W, van der Flier WM, Koene T, Smits LL, Scheltens P, Stam CJ. Disrupted modular brain dynamics reflect cognitive dysfunction in Alzheimer's disease. Neurolmage. 2012;59(4):3085-93.

14. de Haan W, van der Flier WM, Wang H, Van Mieghem PF, Scheltens P, Stam CJ. Disruption of functional brain networks in Alzheimer's disease: what can we learn from graph spectral analysis of resting-state magnetoencephalography? Brain Connect. 2012;2(2):45-55.

15. Li Y, Wang Y, Wu G, Shi F, Zhou L, Lin W, Shen D. Discriminant analysis of longitudinal cortical thickness changes in Alzheimer's disease using dynamic and network features. Neurobiology of Aging. 2012;33(2):427.e415-30.

16. Stam CJ, Hillebrand A, Wang H, Van Mieghem P. Emergence of modular structure in a large-scale brain network with interactions between dynamics and connectivity. Front Comput Neurosci. 2010;4(1):231-6.

17. Tijms BM, Moller C, Vrenken H, Wink AM, de Haan W, van der Flier WM, Stam CJ, Scheltens P, Barkhof F. Single-subject grey matter graphs in Alzheimer's disease. PLoS One. 2013;8(3):e58921.

18. Xie T, He Y. Mapping the Alzheimer's brain with connectomics. Front Psychiatry. 2011;2:77.

19. Wee CY, Yap PT, Shen D. Alzheimer's disease neuroimaging I. Prediction of Alzheimer's disease and mild cognitive impairment using cortical morphological patterns. Hum Brain Mapp. 2013;34(12):3411-25.

20. John M, Ikuta T, Ferbinteanu J. John M, Ikuta T, Ferbinteanu J. Graph analysis of structural brain networks in Alzheimer's disease: beyond small world properties. Brain Struct Funct. 2017; 222(2):1-20.

21. Fischer FU, Wolf D, Scheurich A, Fellgiebel A. Alzheimer's disease neuroimaging I. Altered whole-brain white matter networks in preclinical Alzheimer's disease. Neuroimage Clin. 2015:8:660-6.

22. Gong G, He Y, Chen ZJ, Evans AC. Convergence and divergence of thickness correlations with diffusion connections across the human cerebral cortex. Neurolmage. 2012;59(2):1239-48.

23. Zhao T, Cao M, Niu H, Zuo XN, Evans A, He Y, Dong Q, Shu N. Age-related changes in the topological organization of the white matter structural connectome across the human lifespan. Hum Brain Mapp. 2015;36(10):3777-92.

24. Tijms BM, ten Kate M, Wink AM, Visser PJ, Ecay M, Clerigue M, Estanga A, Garcia Sebastian M, Izagirre A, Villanua J, et al. Gray matter network disruptions and amyloid beta in cognitively normal adults. Neurobiol Aging. 2016;37:154-60.

25. Yao Z, Zhang Y, Lin L, Zhou Y, CI X, Jiang T. The Alzheimer's Disease Neuroimaging Initiative. Abnormal cortical networks in mild cognitive impairment and Alzheimer's disease. PLoS Comput Biol. 2010;6(11): e1001006.

26. Ebadi A, Dalboni da Rocha JL, Nagaraju DB, Tovar-Moll F, Bramati I, Coutinho G, Sitaram R, Rashidi P. Ensemble classification of Alzheimer's disease and mild cognitive impairment based on complex graph measures from diffusion tensor images. Front Neurosci. 2017;11(56):1-17.

27. Wang T, Shi F, Jin Y, Yap PT, Wee CY, Zhang J, Yang C, Li X, Xiao S, Shen D. Multilevel Deficiency of White Matter Connectivity Networks in Alzheimer's Disease: A Diffusion MRI Study with DTI and HARDI Models 2016; 2016:2947136

28. Xie Y, Cui Z, Zhang Z, Sun Y, Sheng C, Li K, Gong G, Han Y, Jia J. Identification of amnestic mild cognitive impairment using multi-modal brain features: a combined structural MRI and diffusion tensor imaging study. J Alzheimers Dis. 2015;47(2):509-22

29. Ghosh A, Deriche R. A survey of current trends in diffusion MRI for structural brain connectivity. J Neural Eng. 2016;13(1):011001.

30. Struyfs H, Hecke W, Veraart J, Sijbers J, Slaets S, De Belder M, Wuyts L, Peters B, Sleegers K, Robberecht C, Broeckhoven CV, De Belder F, Parizel PM, Engelborghs S. Diffusion kurtosis imaging: a possible MRI biomarker for AD diagnosis? J Alzheimers Dis. 2015;48(4):937-48.

31. Jensen JH, Falangola MF, Hu C, Tabesh A, Rapalino O, Lo C, Helpern JA. Preliminary observations of increased diffusional kurtosis in human brain following recent cerebral infarction. NMR Biomed. 2011;24(5):452-7.
32. Wu EX, Cheung MM. MR diffusion kurtosis imaging for neural tissue characterization. NMR Biomed. 2010;23(7):836-48.

33. Stokum JA, Sours C, Zhuo J, Kane R, Shanmuganathan K, Gullapalli RP. A longitudinal evaluation of diffusion kurtosis imaging in patients with mild traumatic brain injury. Brain Inj. 2015;29(1):47-57.

34. Umesh Rudrapatna S, Wieloch T, Beirup K, Ruscher K, Mol W, Yanev P, Leemans A, van der Toorn A, Dijkhuizen RM. Can diffusion kurtosis imaging improve the sensitivity and specificity of detecting microstructural alterations in brain tissue chronically after experimental stroke? Comparisons with diffusion tensor imaging and histology. Neurolmage. 2014;97:363-73.

35. Surova Y, Lampinen B, Nilsson M, Latt J, Hall S, Widner H, Swedish Bio F, van Westen D, Hansson O. Alterations of diffusion kurtosis and neurite density measures in deep Grey matter and white matter in Parkinson's disease. PLoS One. 2016;11(6):e0157755.

36. Bai Y, Lin Y, Tian J, et al. Grading of gliomas by using Monoexponential, Biexponential, and stretched exponential diffusion-weighted MR imaging and diffusion kurtosis MR imaging. Radiology. 2016;278(2):496-504.

37. Gong NJ, Wong CS, Chan CC, Leung LM, Chu YC. Correlations between microstructural alterations and severity of cognitive deficiency in Alzheimer's disease and mild cognitive impairment: a diffusional kurtosis imaging study. Magn Reson Imaging. 2013;31(5):688-94.

38. Gong NJ, Chan CC, Leung LM, Wong CS, Dibb R, Liu C. Differential microstructural and morphological abnormalities in mild cognitive impairment and Alzheimer's disease: evidence from cortical and deep gray matter. Hum Brain Mapp. 2017:38(5):2495-508.

39. Chen Y, Sha M, Zhao X, Ma J, Ni H, Gao W, Ming D. Automated detection of pathologic white matter alterations in Alzheimer's disease using combined diffusivity and kurtosis method. Psychiatry Res. 2017;264:35-45.

40. McKhann G, Drachman D, Folstein M, Katzman R, Price D, Stadlan EM. Clinical diagnosis of Alzheimer's disease: report of the NINCDS-ADRDA work group under the auspices of Department of Health and Human Services Task Force on Alzheimer's disease. Neurology. 2011;77(4):333-3.

41. Tabesh A, Jensen JH, Ardekani BA, Helpern JA. Estimation of tensors and tensor-derived measures in diffusional kurtosis imaging. Magn Reson Med. 2011;65(3):823-36

42. Baser PJ. Inferring microstructural features and the physiological state of tissues from diffusion weighted images. NMR Biomed. 1995;8(7-8):333-44.

43. Hui ES, Cheung MM, Qi L, Wu EX. Towards better MR characterization of neural tissues using directional diffusion kurtosis analysis. Neurolmage. 2008; 42(1):122-34.

44. Clerx L, Visser PJ, Verhey F, Aalten P, New MRI. Markers for Alzheimer's disease: a meta-analysis of diffusion tensor imaging and a comparison with medial temporal lobe measurements. J Alzheimers Dis. 2012;29(2):405-29.

45. Collins DL, Neelin P, Peters TM, Evans AC. Automatic 3D intersubject registration of MR volumetric data in standardized Talairach space. J Comput Assist Tomogr. 1994;18(2):192-205.

46. Tzourio-Mazoyer N, Landeau B, Papathanassiou D, Crivello F, Etard O, Delcroix N, Mazoyer B, Joliot M. Automated anatomical labeling of activations in SPM using a macroscopic anatomical Parcellation of the MNI MRI single-subject brain. Neurolmage. 2002;15(1):273-89.

47. Cui Z, Zhong S, Xu P, He Y, Gong G. PANDA: a pipeline toolbox for analyzing brain diffusion images. Front Hum Neurosci. 2013;7:42.

48. Wang J, Wang X, Xia M, Liao X, Evans A, He Y. Corrigendum: GRETNA: a graph theoretical network analysis toolbox for imaging connectomics. Front Hum Neurosci. 2015;9(386):386.

49. Watts DJ, Strogatz SH. Collective dynamics of 'small-world' networks. Nature. 1998;393(6684):440-2.

50. Maslov S, Sneppen K. Specificity and stability in topology of protein networks. Science. 2002;296(5569):910-3.

51. Sporns O, Zwi JD. The small world of the cerebral cortex. Neuroinformatics. 2004;2(2):145-62.

52. Bullmore ET, Suckling J, Overmeyer S, Rabe-Hesketh S, Taylor E, Brammer MJ. Global, voxel, and cluster tests, by theory and permutation, for a difference between two groups of structural MR images of the brain. IEEE Trans Med Imaging. 1999;18(1):32-42.

53. Mesulam M-M. From sensation to cognition. Brain. 1998;121:1013-52.

54. Daianu M, Jahanshad N, Nir TM, Toga AW, Jack CR, Jr., Weiner MW, Thompson PM, Alzheimer's disease neuroimaging I. Breakdown of brain connectivity between normal aging and Alzheimer's disease: a structural k-core network analysis. Brain Connect. 2013; 3(4):407-422. 
55. Stam CJ, Jones BF, Nolte G, Breakspear M, Scheltens P. Small-world networks and functional connectivity in Alzheimer's disease. Cereb Cortex. 2007:17(1):92-9.

56. Kaustubh Supekar VM, Rubin D, Musen M, Greicius MD. Network analysis of intrinsic functional brain connectivity in Alzheimer's disease. PLoS Comput Biol. 2008;4(6):e1000100.

57. de Haan W, Pijnenburg YA, Strijers RL, van der Made Y, van der Flier WM, Scheltens $P$, Stam CJ. Functional neural network analysis in frontotemporal dementia and Alzheimer's disease using EEG and graph theory. BMC Neurosci. 2009;10(1):101.

58. Stam CJ, de Haan W, Daffertshofer A, Jones BF, Manshanden I. Van Cappellen van Walsum AM, Montez T, Verbunt JP, de Munck JC, van Dijk $\mathrm{BW}$, et al. graph theoretical analysis of magnetoencephalographic functional connectivity in Alzheimer's disease. Brain. 2009;132(Pt 1):213-24.

59. Martín Noguerol T, Martínez Barbero JP. Advanced diffusion MRI and biomarkers in the central nervous system: a new approach. Radiología (English Edition). 2017;59(4):273-85.

60. Steven AJ, Zhuo J, Melhem ER. Diffusion kurtosis imaging: an emerging technique for evaluating the microstructural environment of the brain. AJR Am J Roentgenol. 2014;202(1):W26-33.

61. Glenn GR, Helpern JA, Tabesh A, Jensen JH. Quantitative assessment of diffusional kurtosis anisotropy. NMR Biomed. 2015;28(4):448-59.

62. Cheung MM, Hui ES, Chan KC, Helpern JA, Qi L, Wu EX. Does diffusion kurtosis imaging lead to better neural tissue characterization? A rodent brain maturation study. Neurolmage. 2009;45(2):386-92.

63. Liu Z, Zhang Y, Yan H, Bai L, Dai R, Wei W, Zhong C, Xue T, Wang H, Feng Y, et al. Altered topological patterns of brain networks in mild cognitive impairment and Alzheimer's disease: a resting-state fMRI study. Psychiatry Res. 2012;202(2):118-25.

64. Zhao X, Liu Y, Wang X, Liu B, Xi Q, Guo Q, Jiang H, Jiang T, Wang P. Disrupted small-world brain networks in moderate Alzheimer's disease: a resting-state FMRI study. PLoS One. 2012;7(3):e33540.

65. Oh G, Ebrahimi S, Wang SC, Cortese R, Kaminsky ZA, Gottesman II, Burke JR, Plassman BL, Petronis A. Epigenetic assimilation in the aging human brain. Genome Biol. 2016;17(76):1-11.

66. Yang Z, Chang C, Xu T, Jiang L, Handwerker DA, Castellanos FX, Milham MP, Bandettini PA, Zuo XN. Connectivity trajectory across lifespan differentiates the precuneus from the default network. Neurolmage. 2014;89:45-56.

67. De Santis S, Gabrielli A, Palombo M, Maraviglia B, Capuani S. Non-Gaussian diffusion imaging: a brief practical review. Magn Reson Imaging. 2011; 29(10):1410-6.

68. Rueda-Lopes FC, Hygino da Cruz LC Jr, Doring TM, Gasparetto EL. Diffusionweighted imaging and demyelinating diseases: new aspects of an old advanced sequence. AJR Am J Roentgenol. 2014;202(1):W34-42.

69. Beason-Held LL, Hohman TJ, Venkatraman V, An Y, Resnick SM. Brain network changes and memory decline in aging. Brain Imaging and Behavior. 2017;11(3):859-73.

70. Knyazeva MG, Carmeli C, Khadivi A, Ghika J, Meuli R, Frackowiak RS Evolution of source EEG synchronization in early Alzheimer's disease. Neurobiol Aging. 2013;34(3):694-705.

71. Zhang HY, Wang SJ, Liu B, Ma ZL, Yang M, Zhang ZJ, Teng GJ. Resting brain connectivity: changes during the progress of Alzheimer disease. Radiology. 2010;256(2):598-606

\section{Ready to submit your research? Choose BMC and benefit from:}

- fast, convenient online submission

- thorough peer review by experienced researchers in your field

- rapid publication on acceptance

- support for research data, including large and complex data types

- gold Open Access which fosters wider collaboration and increased citations

- maximum visibility for your research: over $100 \mathrm{M}$ website views per year

At BMC, research is always in progress.

Learn more biomedcentral.com/submissions 